\title{
Defining Early IT System Requirements with Regulation Principles: The Lightswitch Approach
}

\author{
Gil Regev, Alain Wegmann \\ School of Computer and Communication Sciences, Ecole Polytechnique Fédérale de Lausanne, \\ Switzerland \\ \{gil.regev, alain.wegmann\}@epfl.ch
}

\begin{abstract}
In this paper we present the Lightswitch approach, an approach for defining early requirements for enterprise IT systems. Using the approach, engineers can model the way an enterprise regulates its relationships with its environment, identify changing conditions within the enterprise and its environment, and propose options for changing this regulation. The engineers can then define initial IT system goals necessary for the above changes. The use of the approach is shown in the real case of a hospital's sterilization department.
\end{abstract}

\section{Introduction}

Early enterprise IT system requirements focus on understanding the context of use of the IT system. This context represents the problems faced by the enterprise and possible solutions to these problems [4] [7]. Early requirements serve as the basis for defining the detailed requirements for the IT system, often called late requirements [13] [4]. In late requirements the focus is on producing a complete and unambiguous description of the system to be built, whereas in early requirements the focus is on creating an understanding of stakeholders' needs and how these can be addressed with an IT system. Enterprises need to satisfy competing stakeholders' needs with often limited resources. This leads enterprises to create compromises between these competing needs.

Moreover, enterprises evolve in a changing environment, the early requirements phase is the right time for thinking about changes that can or should be made to the enterprise itself [13]. Hence, before an IT system is built, it is important to think about the changing nature of the enterprise and its environment, and to attempt to define early requirements that fit these changes. However, enterprises cannot absorb any amount of change, i.e. they need some stability to be successful. There is therefore a need for methods that encourage innovation while discouraging unacceptable change.

The Lightswitch approach was defined specifically for this purpose. It is built on regulation principles. The main aspect of these principles is the maintenance of identity in a changing environment. The Lightswitch goal-oriented modeling method is derived from these principles. The Lightswitch method can be used to understand who the stakeholders of the enterprise are, how they regulate their relationships (including what compromises they create between the competing demands of stakeholders), what the changing conditions that affect this regulation are, how this regulation may be changed with respect to these changing conditions and what are the resulting highlevel goals for the IT system. It thus can be used to complement existing early or late requirements methods.

In Section 2 of this paper we present the regulation principles that provide the theoretical base for the Lightswitch method. In Section 3 we present the method itself. In Section 4 we illustrate the use of the method with a summary of a case study performed in a major Swiss hospital. In Section 5 we describe the related work.

\section{The regulation principles}

The regulation principles are inspired by General Systems Thinking [11] and Cybernetics [1]. Some of the basic ideas are similar to SSM [2].

The main point of departure for the definition of the regulation principles is to understand how the identity of an enterprise is maintained in an ever changing world. In General Systems Thinking the answer is the "open system" concept. An open system is a system that has relationships with its environment. An open

Presented at the $12^{\text {th }}$ IEEE International Requirements Engineering Conference 2004 (RE’04), September 6-10 
system maintains its internal organization, i.e. its identity, stable by regulating the exchanges it has with its environment. Regulation is the elimination of variety [12]. The result of regulation is the adherence to stable states that we call norms. When the state of affairs is considered to deviate substantially from the norm (i.e., to be outside of an acceptable threshold), a regulative action may be taken to bring the state of affairs closer to the norm or the norm may be changed or both [9].

Since the environment changes continuously, the system needs to adapt to it from time to time. Thus, the system changes over time. Through the regulation of its relationships, the system attempts to limit these changes. In other words, regulation seeks to maintain the state of a variable within some bounds. The success of this regulation leads to the maintenance of identity of the system and therefore to its survival. Hence, we consider that systems survive in a changing environment by maintaining some of their norms unchanged and changing others to adapt to the changing environment.

We call interpretation, a system's understanding of itself and its environment. Norms influence each other through each system's understanding of these norms, i.e. through the system's interpretation of the norms. Thus, different systems can have different interpretations of a same norm or a given system may change its interpretation of a norm. Therefore, for a system to change its norms, its interpretations need to change as well. Also, regulative action is taken with respect to interpretations of what constitutes a deviation. In other words, different worldviews lead to different behaviors.

The Lightswitch regulation oriented heuristics can be used to analyze an enterprise's business processes and infer how the enterprise regulates its relationships with its stakeholders.

\subsection{The regulation oriented heuristics}

The heuristics are derived from Weinberg and Weinberg's analysis of regulation in systems [12]. In the following list we mention only a few of the heuristics from [6].

\section{Specialization:}

Providing specific mechanisms for regulating a subset of norms. Specialization provides a better regulation for a given set of norms but reduces the ability to regulate other norms.

\section{Ample and scarce resources:}

Access to scarce resources is highly regulated. Access to ample resources is much less regulated.
Some resources are so ample that they become hidden and access to them is not regulated at all. Scarce resources may become ample and ample resources may become scarce. This causes instability in systems that need to adapt to such changes.

\section{Compromises and dissatisfaction:}

Compromises are necessary when a system needs to regulate several relationships at the same time. Compromises do not satisfy stakeholders completely, leading to dissatisfaction.

\section{Backup regulation mechanisms:}

Mechanisms that are used to maintain a state of affairs within the acceptable threshold of the norm even when the main mechanism fails to do this due to unexpected changes

\section{Regulation by error:}

Waiting for a deviation from the norm to be out of the acceptable threshold before taking regulative action.

\section{Regulation by anticipation:}

Taking regulative action before a deviation from a norm is out of the acceptable threshold. When anticipation succeeds it provides a better regulation that through error by reducing the deviation from the norm. When incorrectly anticipating a deviation, the result may be significantly less satisfactory. Anticipation requires experience.

\section{The Lightswitch method}

The Lightswitch method is built on the basis of these regulation principles. It is composed of two main parts: the Lightswitch models and the process used to structure the intervention in an enterprise. We review each one of these parts in turn.

\subsection{The Lightswitch models}

The Lightswitch models use standard goal-oriented modeling elements such as maintenance and achievement goals [3], beliefs [13], and communities that are similar to an agent. The correspondence between the modeling elements and the regulation principles are:

- a Community represents a system

- a Belief within a community represents an interpretation of the system

- a Maintenance goal within a community represents a norm of the system

- $\quad$ an Achievement goal of a community represents a regulative action the system 
The notation used to represent the Lightswitch models is based on the Unified Modeling Language (UML) [5].

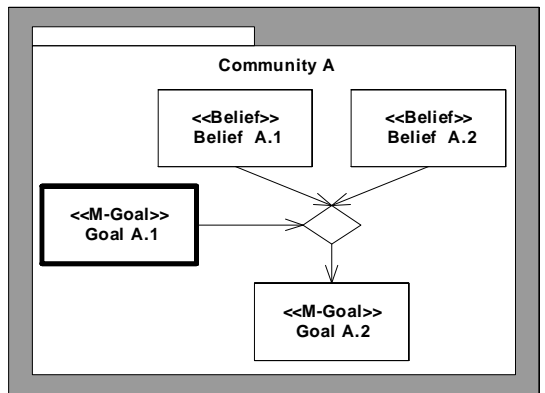

Figure 1. A sample model of a community

Figure 1 shows a first sample model of a community. In this figure we can see that:

- Communities are represented as UML packages with the name of each community displayed at the top.

- The shaded rectangle around the community represents the environment of the community that is not considered in the model.

- Maintenance goals, achievement goals, and beliefs are represented as UML classes within communities. They are distinguished through the use of UML stereotypes, i.e., a maintenance goal is designated by $<<\mathrm{M}$ Goal $>>$, an achievement goal by $<<\mathrm{A}-$ Goal $>>$, and a belief by $<<$ Belief $>>$.

- Relationships are represented with UML associations.

- UML N-ary associations are used to represent multiple relationships between beliefs and goals. In Lightswitch, $\mathrm{N}$-ary associations are used specifically to represent goal reduction, i.e., showing how one or more goals are related to one or more sub-goals through the consideration of one or more beliefs. The higher-level goal is the goal that is represented as an input into the n-ary association (appears in bold in Figure 1). The lower level goals are represented as results of the n-ary association.

The N-ary association represents what is known in goal-oriented RE literature (e.g. [3]) as an "and" relationship among the subgoals. For an "or" relationship to be represented, the beliefs need to be changed and hence a different model needs to be created. As an example, consider the goal of making a purchase. Two subgoals with an OR relationship may be to "pay cash" or "pay with a credit card." Paying with a credit card may be justified in a Lightswitch model with the following belief, "paying with a credit card allows me to keep more cash in the bank." To justify paying cash, a different belief is needed in the model, such as, "I will need to pay interest if I pay with a credit card." We thus need to make two different models, containing a different set of beliefs, to express these two different subgoals.

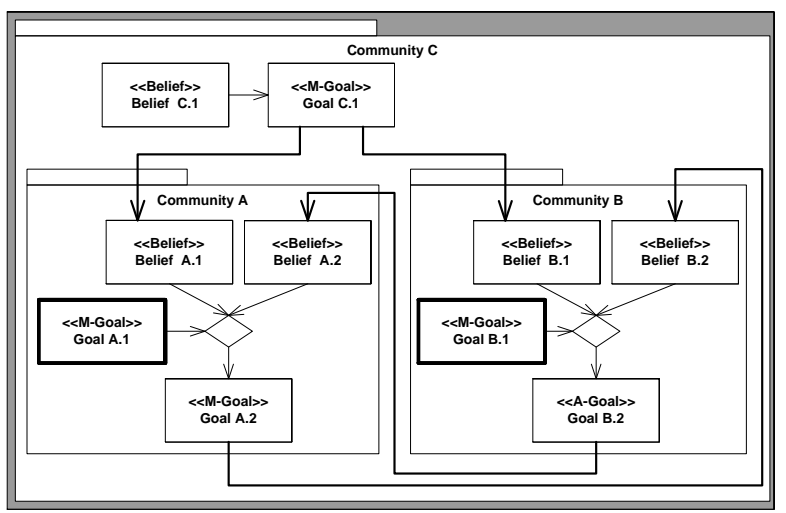

Figure 2. A sample interaction of two communities within a supra-community

Community A and Community B can be modeled within the larger context of the supra-community $\mathrm{C}$ (Figure 2). With respect to community C, Community $\mathrm{A}$ and Community B are sub-communities. Community $\mathrm{C}$ also has a belief and a maintenance goal. The maintenance goal Goal C.1 influences both Community A and Community B by being linked to Belief A.1 and Belief B.1.

The reader might have noticed that we use a very limited set of concepts: systems, norms, regulative actions, and interpretations that are modeled with communities, maintenance goals, achievement goals, and beliefs respectively. This sparseness of concepts was a design goal of the Lightswitch method. This was based on the assumption that a small set of concepts is more appropriate for an early requirements method where ease of use, ease of understanding, and flexibility are very important. The drawback of this approach is that there is less richness available to describe a situation. For example, some people may argue that some issues that we describe as norms (for example, the rise in healthcare costs in the case study) are in fact trends. Also, some aspects that we model as beliefs would be referred to by many people as facts. Here the issue is not as much an issue of sparseness of concepts as it is an issue of dealing with a changing world. In a changing world, facts have a tendency to change. Modeling a system's understanding of its environment as beliefs rather than facts may help stakeholders to explore different models containing different beliefs. 


\subsection{The Lightswitch process}

The Lightswitch process consists of 4 major steps. The process is usually performed iteratively, each step being performed up to a point where it yields a model that is good enough to perform the next step. The steps can be repeated as necessary until the resulting model is judged to be of sufficient quality.

Step 1: Identification of stakeholders

This step consists in identifying nouns in enterprise discourse and in modeling enterprises as communities within communities. Engineers are encouraged to go beyond the immediate preoccupations of the stakeholders and identify the environment of the enterprise at the highest level of abstraction possible. This often leads to the modeling of the environment of the environment etc. of the enterprise.

\section{Step 2: Analysis of current regulation}

This step consists in analyzing how the enterprise regulates its relationships with its stakeholders. The analysis is performed by modeling the relationships with maintenance goals and beliefs within the communities identified in step 1 and by analyzing the business processes of the stakeholders with the regulation oriented heuristics described above.

This step may include the identification of stakeholders that are not named in the discourse but whose existence can be inferred by understanding how the relationships are regulated.

Step 3: Analysis of the adequacy of the regulation

This step consists in identifying conditions within the enterprise and its environment that have rendered or may render the current regulation inadequate. This step includes the comparison of current conditions with past conditions, as well as forecasting future conditions. As such, it is obviously subject to the impossibility of completely knowing the past and of forecasting the future. The regulation heuristics are again used as guides in understanding how the regulation may be changing.

Step 4: Proposing changes to regulation and specifying IT system goals

This step consists in identifying and evaluating options followed by a selection of the most viable option, considering the existing and foreseeable norms. This step includes an analysis of compromises needed to satisfy the expectations of stakeholders (quality of service), the available resources, and the need to maintain stability. The regulation heuristics are used to analyze the consequences of the different options on the stakeholders.
Following the selection of an option, we derive IT system goals for this option.

In the following section we show how the Lightswitch method can be applied to a real-world requirements engineering effort.

\section{The Hospital Case Study}

The CHUV (Centre Hospitalier Universitaire Vaudois) is the main hospital in the Canton of Vaud, Switzerland. A particular preoccupation in every hospital is the absence of germs of any kind in the medical equipment that is used to care for patients. This medical equipment consists of anything that comes into contact with patients, bandages, needles used for injections, instruments used in operations, implants such as screws, etc. The CHUV, as most hospitals, has a sterilization department. The sterilization department sterilizes medical material used by the other departments of the hospital. The sterilization department uses an IT application called Mikros to help it with some of its operations.

Mikros has been in use for about 15 years and is now regarded as obsolete. It has been decided that it can no longer be maintained and that it needs to be changed. A team of three people, the manager of the sterilization service, a software project manager and a software team manager were asked to conduct an "opportunity study." The aim of this study was to define the needs of the sterilization department in terms of IT systems for the 2002-2005 timeframe. These needs should be used for the early requirements a future IT system. The study was conducted from April to July 2002. The study included interviews of several people in high management positions within the hospital, several debriefing sessions, visits to the sterilization department, and a visit to the sterilization service of a French hospital using a state of the art IT system.

The way the study was organized, showed that the problem of the replacement of Mikros was not only analyzed from the point of view of what Mikros does today and how to replace it with a state-of-the-art system but also included an analysis of the global context up to the strategic changes expected in the Swiss healthcare landscape and the needs of the clients of the sterilization department. In the following subsections we show how organizational changes can be proposed and evaluated and how early requirements can be defined by following the Lightswitch method. This is a condensed version of the case that was published in [6]. 
All models and discussions in this paper represent our interpretations and do not represent an official position of the hospital.

\subsection{Identification and analysis of stakeholders (step 1)}

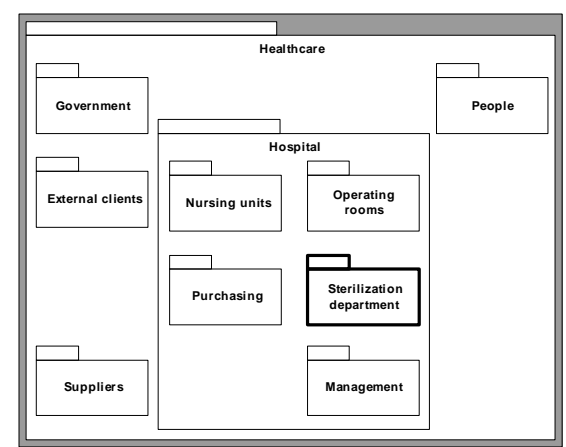

Figure 3. Initial context diagram

The results of the interviews enabled us to define the initial context diagram in Figure 3 that shows the sterilization department and its environment as communities. As specified in this step, we attempt to define the highest level of abstraction. We thus model not only the immediate environment of the sterilization department, i.e. the hospital but also the environment of the hospital itself, i.e., the Swiss healthcare environment.
We model this environment as communities, such as people and government. We place both communities in a community we can refer to as the healthcare community.

\subsection{Analysis of current regulation (step 2)}

The issues that were raised most often by the stakeholders were the new norms defined by the Swiss government requiring the traceability of medical equipment, the distribution of medical material to internal clients and the need to control costs in general and the cost of inventory in particular. In this paper we will focus on analyzing the inventory problem.

Some of the relevant norms that can be identified in the environment of the hospital are:

- the continuous rise in health care costs

- the continuous improvement in the quality of healthcare

- people's desire for quality healthcare

- people's desire for improvement of this quality

- people's desire for a limitation of healthcare cost

These norms result in pressure being put on the government to require healthcare providers to limit costs, maintain and improve the quality of healthcare. These norms are modeled in Figure 4 as maintenance goals connected through their associated beliefs. The highest level goals found in this model are for the different communities to maintain their mutual relationships.

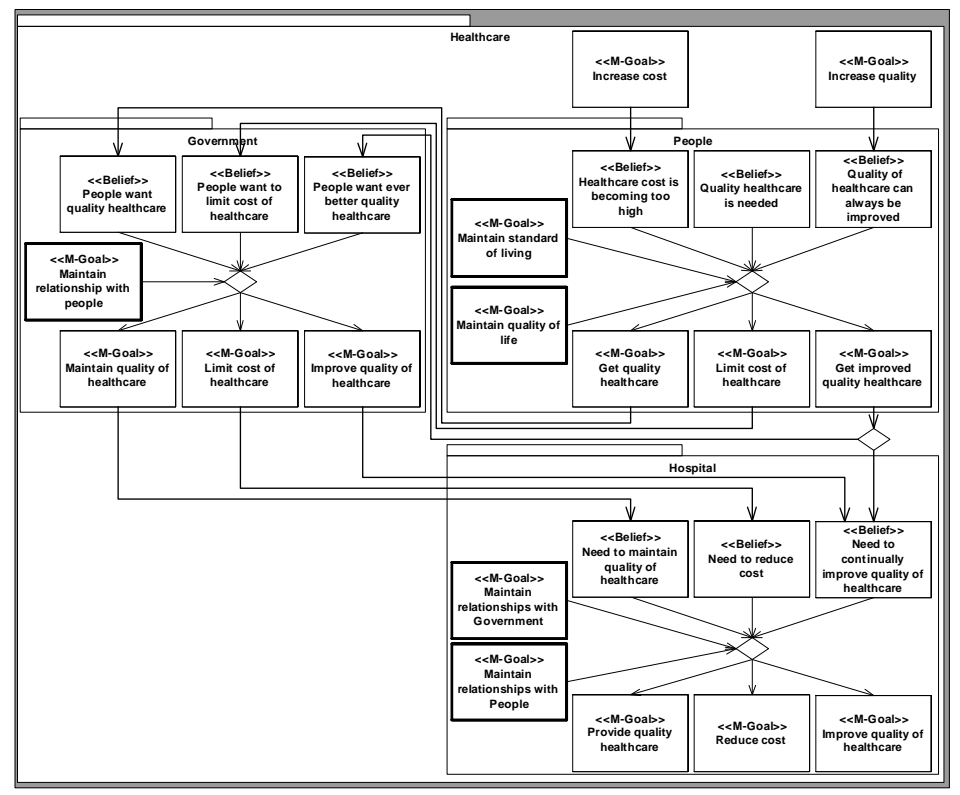

Figure 4. The environment of the hospital 
To limit healthcare costs the resources provided to hospitals are limited. To provide better quality with fewer resources, access to scarce resources (such as money, medical material, and people) is closely regulated, as suggested in the previous section. This regulation puts into motion the scrutiny of existing business processes and therefore gives a context to the issues raised by the interviewees.

In order to understand the role of the sterilization department within the hospital, we can model why it exists in the first place, we can begin with the goal identified in Figure 4, "Provide quality healthcare." When the hospital was created, a sterilization department was created on site and given the responsibility to sterilize medical material used to provide care. This was done because a subgoal of the provision of quality healthcare is to avoid nosocomial infections.

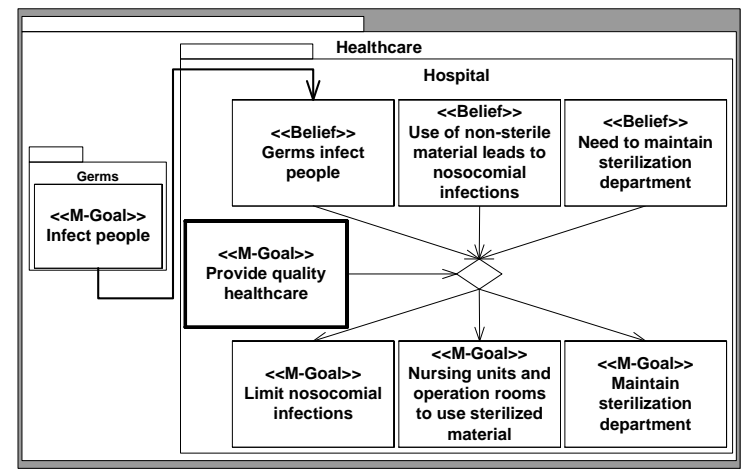

\section{Figure 5. Reasons for maintaining a} sterilization department

At the same time, a norm was set that specifies that care be only provided with sterilized material. This is modeled in Figure 5. Over the years, the sterilization department has become an integral part of the operations of the hospital. Indeed, the nursing units became dependent on the ability of the sterilization department to remove used material and for the timely provision of sterilized, adequate material. This distribution of work has become the norm in the hospital. This situation is modeled in Figure 6 where the sterilization department has the goal of maintaining relationships with nursing units and the nursing units have the goal of providing care to patients.

Analyzing the sterilization department's business processes enables us to understand how the relationships between the sterilization department and some of its clients, such as the nursing units, are regulated. For example, the process that we could call delivery of sterilized material to nursing units specifies several norms, two of which are:
1. The sterilization department is responsible for removing used material from the nursing units, sterilizing the material and returning it to the nursing units.

2. There is a distinction between material that is specific to a given nursing unit and material that is generic, i.e. used in several units. Specific material is owned by the nursing unit and should be returned once sterilized.

Generic material is sterilized then stored in the sterilization department warehouse and is distributed to nursing units upon request.

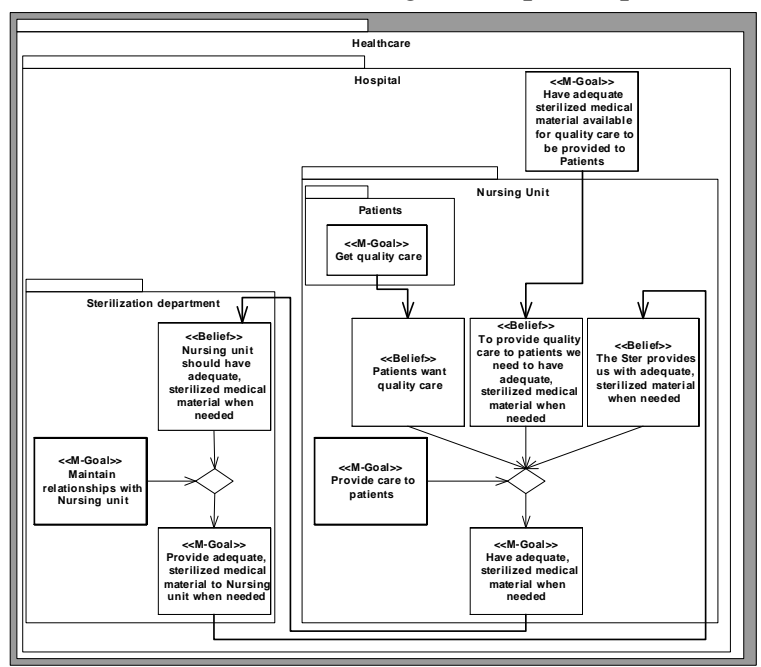

Figure 6. Relationships between sterilization department and nursing units

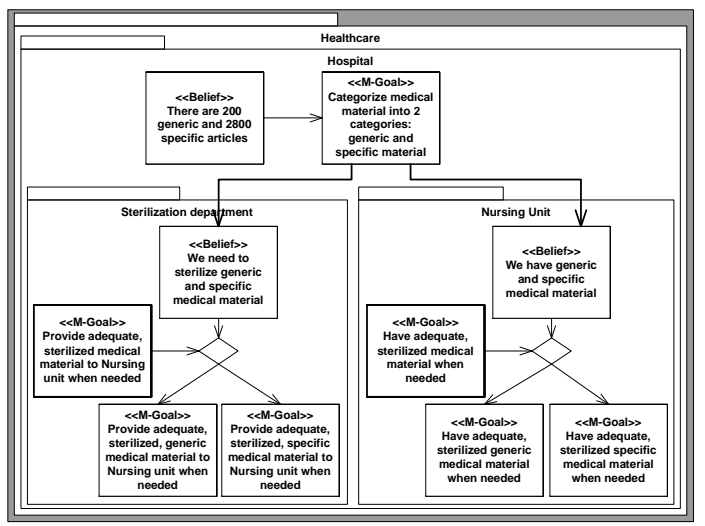

Figure 7. Delivery of material to nursing units

Figure 7 models the norm that distinguishes between specific and generic material, taking as a point of departure the goals for the provision of medical material identified in Figure 6. This distinction has resulted in the sterilization department's specialization to these two norms. Indeed the department has two specialized processes, one for the treatment of specific 
material and the other for generic material. We will now focus on the generic material process.

\subsection{Analysis of the adequacy of the regulation (step 3)}

The model in Figure 8 provides a further analysis of the distribution of generic material to nursing units. The model shows a modus vivendi between the sterilization department and nursing unit regarding the management and distribution of generic material.

The maintenance of a minimum of inventory by the typical nursing unit is necessary for it to maintain its capability to deliver care to patients. Indeed, the nursing unit is physically distant from the sterilization department, and it faces unpredictable situations with patients. It thus cannot rely on the sterilization department's inventory for its day to day operations but needs to maintain its own inventory.

The existence of the nursing unit inventory, however, creates a situation where the sterilization department can make partial deliveries. A partial delivery is made when the sterilization department inventory is out of stock. The existence of the concept of partial delivery and the existence of the nursing unit's inventory are interdependent. The Maintenance of inventory by the nursing unit is interpreted by the sterilization department as relieving itself from managing its inventory too closely. In terms of the Lightswitch heuristics, this corresponds to a regulation by error. At the same time, the occurrences of partial deliveries encourage the nursing unit to protect its ability to deliver care to patients by maintaining a relatively large inventory and by ordering medical material preventively. In terms of the Lightswitch heuristics this corresponds to the regulation by anticipation. Thus, this situation probably leads to a large inventory in the nursing unit, which anticipates the sterilization department's inability to deliver complete orders in order to protect themselves from partial deliveries. Note that this was identified as a potential problem in the regulation by anticipation heuristic.

Another factor, shown in Figure 8, that contributes to large nursing unit inventory is the nursing unit's belief: "We don't have time and knowledge required to manage our inventory.” This may be caused by a high turnaround rate of personnel in nursing units, the nursing units' personnel being preoccupied with more urgent tasks such as caring for patients, and nursing units' lack of inventory management tools. Furthermore, each nursing unit probably spends some of its time managing its inventory, which can be seen as not being one of its primary duties.

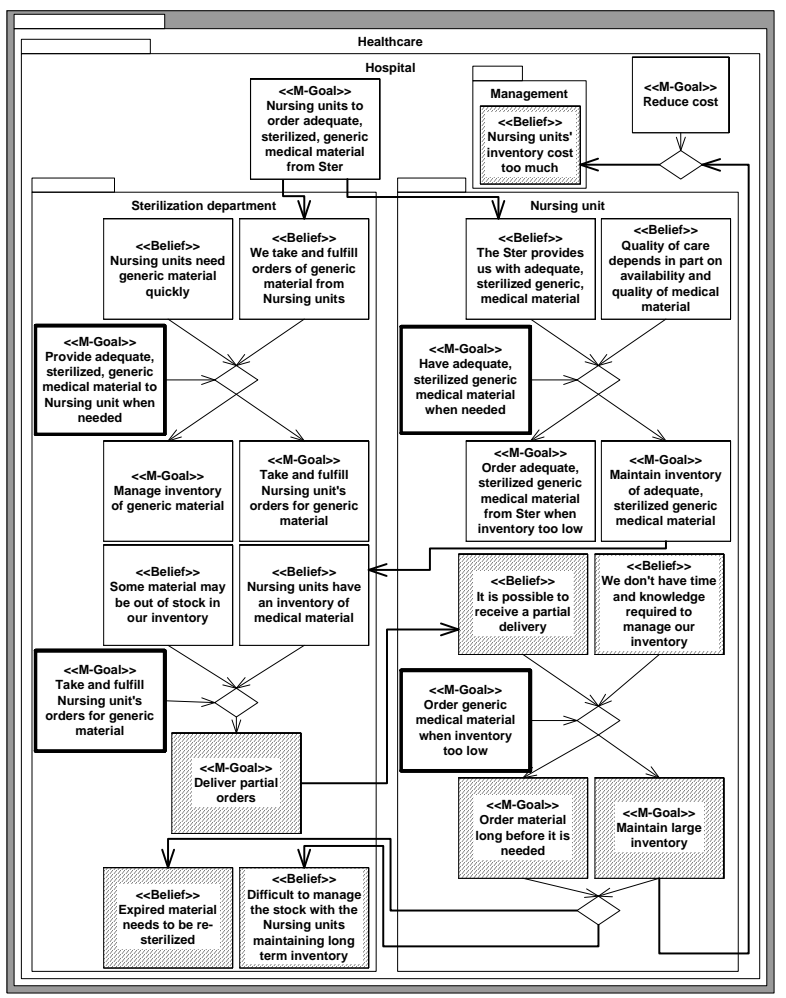

Figure 8. Reasons for the inventory problem

The beliefs about this situation by the different communities involved are the following:

- The sterilization department believes that this leads to waste due to expiry dates on sterilized material which leads to either throwing away of material or re-sterilization of expired material.

- The sterilization department believes that it cannot correctly manage its inventory, i.e., due to the accumulation of material in the nursing units, the sterilization department cannot predict what levels of inventory it needs to have. Thus, for the sterilization department, partial deliveries may happen because of the large nursing units' inventories. We are in a self perpetuating cycle.

- Management believes that this is an unsatisfactory situation with respect to the cost of inventory. Hence, the environment of the sterilization department is changing. A situation that was accepted before is now viewed as a problem.

In terms of the Lightswitch heuristics these beliefs correspond to stakeholders' dissatisfactions. 
The people we interviewed in the sterilization department believe that the issue of partial deliveries was relevant in the past but that now they manage their inventory in a way that insures complete deliveries. If this is the case, then we can see that the nursing units are probably anticipating on a problem that does not exist anymore. However, since being out of stock represents a much more important threat to their ability to provide care than the existence of too much inventory, they are willing to sacrifice some inefficiency in their use of resources rather than lose the ability to correctly regulate their relationships with their own clients, i.e. their patients.

\subsection{Proposing changes to regulation and specifying IT system goals (step 4)}

We can think of several optional courses of action in this situation. Any of these proposed options may encounter forces that act against change as part of the hospital and its constituents' maintenance of their identity. This is known as the homeostatic principle [12] [6]. In the following list we describe two of these options and explain some of their implications:

Option 1: Maintain the nursing units' inventories but make the sterilization department responsible for managing it. This places the inventory regulation factor solely in the sterilization department. The sterilization department will have the goal of maintaining adequate inventory levels in the nursing units.

This solution would require the sterilization department to build statistics on which nursing unit uses what material and at what rate, and use these statistics to predict the needs of each nursing unit in order to maintain its inventory.

A backup mechanism will be needed, i.e., an emergency delivery service to nursing units in case they are out of stock due to an unanticipated surge in its activities. In terms of the Lightswitch heuristics, this mechanism is an anticipation of future problems.

This solution will remove the responsibility of the nursing units to manage their inventory, thus freeing it to focus on the provision of care to patients. The nursing units will then depend even more on the sterilization department's ability to manage the inventory, i.e., the nursing units will lose some of their autonomy.

The sterilization department will need to have more personnel and train them to manage the nursing unit inventory.

In terms of the Lightswitch heuristics, the sterilization department will be more specialized toward nursing units. The solution may be more efficient but will result in an increased interdependence between nursing units and sterilization department.

Option 2: Require the nursing units to reduce its inventory to a minimum and rely on the sterilization department's ability to do fast and complete deliveries. This maintains the inventory regulation factor in the nursing units but also requires the sterilization department to anticipate the aggregated consumption of all nursing units.

The sterilization department will have the goals of maintaining its inventory level so as not to run out of stock and insuring delivery of orders before nursing units run out of their minimal inventory.

The nursing units will retain their autonomy, i.e., the responsibility and capability of managing their inventory. On the other hand, it will be taking responsibilities that may be out of its scope of providing care to patients. So in situations of stress, the quality of the inventory management may drop. This option may also cost more (at least for the short term) than option 1 , because it may cost more to train all the nursing units' personnel to manage their inventory than to train the sterilization department's personnel only.

These options represent different compromises in the use of scarce resources (e.g. medical material, financial resources) between specialization and autonomy; between regulation by error and by anticipation. These compromises have different consequences for the relationships (e.g. level of service furnished) between the sterilization department and nursing units on one hand the nursing units and clients on the other hand.

Figure 9 shows the model for option 1. The belief of management that the nursing units' inventory is too expensive and the belief that the nursing units cannot reliably manage their inventory now result in a maintenance goal that instructs the sterilization department to manage the nursing units' inventories.

By placing these beliefs and this maintenance goal in the hospital community, we wish to show that this has become the norm of the whole hospital.

This maintenance goal influences both the nursing unit and the sterilization department. The nursing unit now relies on the sterilization department's ability to manage its inventory. A backup regulation mechanism for urgent orders is provided to handle unexpected events in the nursing units. Notice that compared to the model in Figure 8 the nursing unit does not have the goal to manage its inventory but the sterilization department's goal has changed from managing its 
inventory to managing the nursing unit's inventory. This means a definite shift in the mission of the sterilization department, which may now focus more on its clients needs.

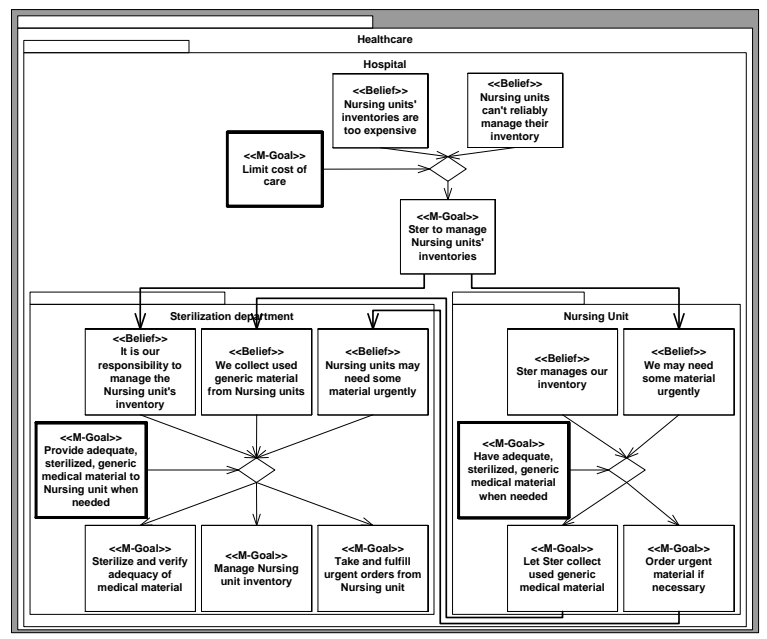

Figure 9. Model of option 1

We now want to analyze what the goals of the IT system should be for option 1 . Figure 10 shows a further reduction of the maintenance goal of the sterilization department, "Manage Nursing unit inventory" and the resulting IT system goals.

The IT system's main goals are shown at the bottom of Figure 10. They are stated as goals that help the sterilization department's personnel to achieve the goals identified above.

In this section we have shown how the Lightswitch method can be used to define early IT requirements for the sterilization department. At first, we did not focus on the sterilization department but on the environment of the hospital. This enabled us to understand some of the norms that apply to the hospital and to the sterilization department. We analyzed the relationships regulated by the sterilization department. We have identified some problems in these relationships, which arise from past norms within the department and in its environment as well as the norms identified earlier in the hospital's environment. We have proposed two optional courses of action for resolving these problems. We have analyzed some of the consequences of the options and defined high-level goals for IT system for one of these options.

One of the results of this phase is to show that the mission of the sterilization department, from the point of view of the hospital, can be seen as "remove and provide sterilized material to nursing units when needed, at the right quality and cost." This relatively large mission may be overlooked if the sterilization department is considered without reference to its norms and those of its environment.

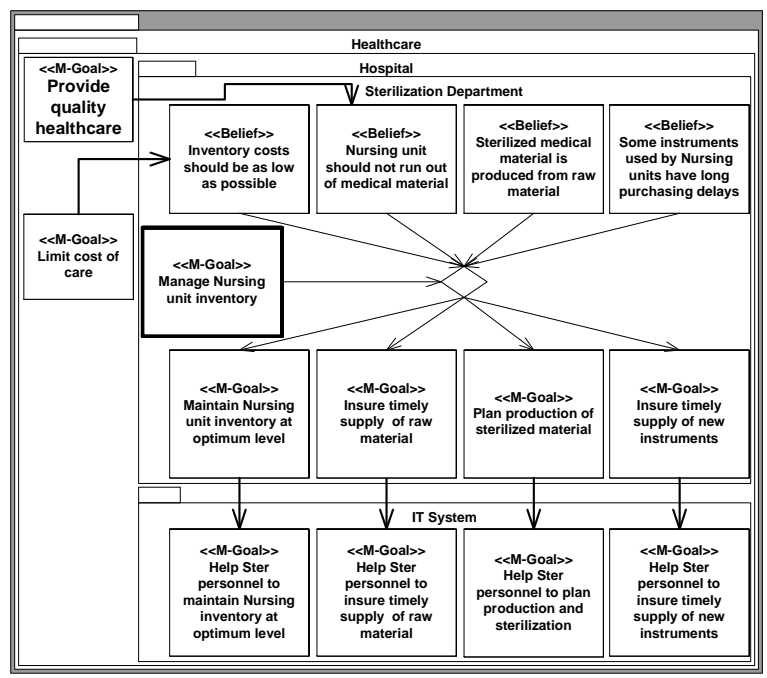

Figure 10. IT system goals for option 1

\section{Related Work}

Early requirements can be seen as the phase where business needs meet the IT system requirements. As such, many methods that have originated at the frontier between business and IT can be used, such as Soft Systems Methodology [2] and Systems Diagrams [8]. The Lightswitch method shares some of the characteristics of these methods, as well as their origins, in the system sciences. However, the Lightswitch method provides more tools for understanding the regulation of relationships from the point of view of the maintenance of identity and it is more strongly coupled with requirements engineering practices, providing specific high-level goals for the envisioned IT system.

In the requirements engineering field, the Volere process' blast off phase [7] provides guidance for the early requirements phase. However, it relies on a well defined problem statement obtained from stakeholders in order to define the purpose and context diagram that can be seen as representing the early requirements. When the problem is not clearly defined, which is often the case in IT projects, defining the system's purpose becomes a project in itself. The Lightswitch approach can help in understanding the problem and defining the purpose.

The $i^{*}$ framework [13] was developed specifically for the early requirements phase. It models an organizational context, such as an enterprise, as strategic relationships between actors that depend on each other for achieving goals, performing tasks, and 
exchanging resources. The Lightswitch method provides an explanation on why these relationships are maintained in certain states. The regulation heuristics enable requirements engineers to understand some of the changes that are occurring in these relationships. Hence, the Lightswitch method can be used as a complement to $i^{*}$ to foster discussion between stakeholders about the way they regulate their relationships, the different courses of action available to them and the influence of these on the IT system requirements.

Furthermore, Lightswitch is a lighter weight method than existing goal-oriented methods, it contains less modeling elements and therefore less symbols to understand and remember.

Lightswitch is more informal than existing methods. This can be seen as a disadvantage for late requirements but may be an advantage when it comes to early requirements where the focus is on specifying high-level goals for the IT system rather than producing accurate and complete requirements.

The Lightswitch concepts bear resemblance with some of the concepts in Wand and Weber's ontology [10]. Whereas a full comparison with this ontology is out of the scope of this paper, it is worthwhile to mention that Wand and Weber define the concepts of stable and unstable states of a system. For Wand and Weber the difference between stable and unstable states relates to the kind of event that can change the state. In Lightswitch the concept of a norm (a stable state) refers to a state that remains relative unchanged in a given period of observation, regardless of the events that may change the state. Furthermore, in Lightswitch we establish a direct relationship between the need to maintain stable states (in changing world) and actions. Wand and Weber do not make this connection.

\section{Conclusions}

The early requirements phase for an enterprise IT system is concerned with setting the main directions for which this system will be built. This phase includes activities for understanding the organizational context of the IT system, i.e., the enterprise. The Lightswitch method, presented in this paper, provides tools for understanding this context by encouraging project participants to model how the enterprise regulates its relationships with its stakeholders. During this analysis the changing nature of the enterprise and its environment, as well as the aspects that the enterprise attempts to maintain unchanged, are analyzed. The result of the analysis is a set of interlinked models that describe how the enterprise maintains its identity in its environment, models of the changing nature of the enterprise and its environment, a set of options to adapt the enterprise to these changing conditions, and initial IT system goals for these options. Future work may consist of the development of a tool that makes the management of Lightswitch models easier in practice.

\section{References}

[1] Ashby, W., R., An Introduction to Cybernetics. Chapman Hall. London, 1956.

[2] Checkland, P. and Scholes, J., Soft System Methodology in action, Wiley, Chichester UK, 1990.

[3] Dardenne, A., van Lamsweerde A. and Fickas, S., "Goal Directed Requirements Acquisition”, Science of Computer Programming, Vol. 20, No. 1-2, pp. 3-50, 1993

[4] Mylopoulos, J., Kolp, M., and Castro, J. "UML for Agent-Oriented Software Development: The Tropos Proposal,” Proc. UML 2001.

[5] OMG Unified Modeling Language (UML) specification version 1.5, march 2003.

[6] Regev G., A Systemic Paradigm for Early IT System Requirements Based on Regulation Principles: The Lightswitch Approach, Ph.D. Thesis, Ecole Polytechnique Fédérale de Lausanne (EPFL), Lausanne, Switzerland, 2003.

[7] Robertson, S. and Robertson, J., Mastering the Requirements Process, Addison-Wesley, Reading MA, 1999. [8] Senge, P., Roberts, C., Ross, R., Smith, B., Roth, G. and Kleiner, A., The Dance of Change, Nicholas Brealey, London, 1999.

[9] Vickers, Sir G., Policymaking, Communication, and Social Learning. Transaction Books. New Brunswick NJ, 1987.

[10] Wand, Y. and Weber, R., "On the deep structure of information systems", Journal of Information Systems, Blackwell Science, 1995, pp. 203-223.

[11] Weinberg, G. M., An Introduction to General Systems Thinking. Wiley \& Sons. New York, 1975.

[12] Weinberg, G. M. and Weinberg, D., General Principles of Systems Design. Dorset House. New York, 1988.

[13] Yu, E.S.K. "Towards modelling and reasoning support for early-phase requirements engineering," Proceedings of the Third IEEE International Symposium on Requirements Engineering, Annapolis, Maryland, January 1997. 